\title{
Laboratory Specimen Type
}

National Cancer Institute

\section{Source}

National Cancer Institute. Laboratory Specimen Type. NCI Thesaurus. Code C83315.

The type of a material sample taken from a biological entity for laboratory testing. 